Somatostatin analogs (SSAs), including lanreotide, play a fundamental role in treatment of neuroendocrine tumors (NETs) of the gastrointestinal tract. SSAs control the clinical symptoms and are the treatment of choice in functioning NETs. Data indicating that SSAs have anti-proliferative activity has mainly come from prospective or retrospective observational studies. A recently published CLARINET study confirmed the anti-proliferative effect of lanreotide in a much broader range of NET patients than previously reported. As a result, it is now possible for clinicians to use lanreotide to treat patients with well-differentiated metastatic grade 1 and grade 2 GEP NETs (i.e., with a Ki-67 proliferative index $<10 \%$ ) located in the pancreas, small intestine, or of unknown primary location, regardless of the degree of liver involvement. The results of the CLARINET study also challenge the current "wait and watch" strategy for NET treatment. Instead, it is proposed that SSAs are considered at an early stage of NET management, as already suggested by many organizations and scientific societies.

Key words: CLARINET, lanreotide, neuroendocrine tumors, somatostatin analogues.

Contemp Oncol (Pozn) 2015; 19 (5): 345-349 DOI: $10.5114 /$ wo.2015.56006

\section{Treatment of neuroendocrine tumors: new recommendations based on the CLARINET study}

\section{Beata Kos-Kudła}

Division of Endocrinology, Department of Pathophysiology and Endocrinology, Medical University of Silesia, Katowice, Poland

Somatostatin analogs (SSAs) play a fundamental role in the diagnosis and treatment of gastroenteropancreatic (GEP) neuroendocrine tumors (NETs) and are the treatment of choice in functioning NETs [1, 2]. SSAs control the clinical symptoms of GEP-NETs caused by excessive tumor secretion of hormones and other biologically active substances. They have multidirectional beneficial effects on the digestive system, including: inhibition of pancreatic and gastrointestinal hormone (insulin, glucagon, gastrin, secretin and vasoactive intestinal hormone) secretion; reduction in intestinal motility and transport; reduction of blood flow in visceral vessels; and inhibition of tissue growth and differentiation.

SSAs exert their biological function by binding with varying affinity to five somatostatin receptors (SST, to $\mathrm{SST}_{5}$ ) present on tumor cell membranes; however, type 2 and type 5 SSTs are mainly expressed on NETs [1, 2]. The antitumor effects of SSAs can be direct, via the interaction with somatostatin receptors, or indirect, through inhibition of growth factor section, immune system modulation, apoptosis induction, and angiogenesis inhibition [3].

Data indicating that SSAs have anti-proliferative activity have mainly come from prospective or retrospective observational studies. These data have so far come only from clinical studies of lanreotide and octreotide. The new SSA pasireotide is under investigation as antitumor therapy, but its efficacy in this indication has not yet been proven [4]. In 2009, the first controlled randomized trial on the antitumor activity of SSAs was performed (the PROMID study). This study demonstrated that the SSA octreotide had anti-proliferative activity in 85 patients with grade 1 (i.e., Ki-67 proliferative index below 2\%), functioning and nonfunctioning midgut NET and with a low hepatic tumor load (metastatic lesion involvement was not greater than 10\%) [5].

More recently, a detailed investigation of the beneficial effect of lanreotide at a dose of $120 \mathrm{mg}$ once every 4 weeks was performed on a broader range of patients. The Controlled Study of Lanreotide Antiproliferative Response in Neuroendocrine Tumors (CLARINET) study was an international, randomized, phase III, placebo-controlled, double-blind trial, which evaluated the use of the long-acting SSA lanreotide Autogel over 96 weeks [6]. Over 200 patients with advanced, highly and moderately differentiated, nonfunctioning grade 1 or 2 (i.e., Ki-67 proliferative index below 10\%) GEP NETs were included in the study. In these patients, the NETs were derived from the pancreas, middle or posterior gut or their primary site was unknown. All NETs expressed somatostatin receptors. Patients with locally advanced, unresectable or metastatic NETs were included in the study, but patients who were treated with interferon, chemoembolization methods, or chemotherapy within the 6 months prior to the study were excluded. In addition, patients who had previously been treated at any time with radioisotopes or SSAs (unless the SSA use was more than 6 months prior to the study) were 
excluded. Patients were randomly assigned to groups receiving 120 mg lanreotide (101 patients) or placebo (103 patients) once every 28 days (Table 1 ).

The primary endpoint was progression-free survival (PFS), defined as time to disease progression (according to the Response Evaluation Criteria in Solid Tumors, version 1.0) or death. PFS was also examined in predefined subgroups, which were selected based on the origin of the primary tumor, tumor grade and hepatic tumor involvement.

\section{Secondary endpoints included:}

- PFS at 12 and 24 months,

- overall survival (defined as the time from randomization to death from any cause),

- time to tumor progression (TTP),

- quality of life (assessed on the basis of questionnaires of the European Organization for Research and Treatment of Cancer - QLQ-C30 and QLQ-GI.NET21),

- the percentage of patients whose plasma chromogranin A decreased by $\geq 50 \%$ from the first measurement to the last available measurement,

- pharmacokinetics,

- safety [6].

From a clinical point of view, it is important to note that $84 \%$ of the NET patients in the study had not been treated previously. In addition, in $96 \%$ of the NET patients, there was no disease progression for 3-6 months prior to randomization, which means that the majority of those patients had stable disease. The primary tumor site is also important for the ongoing management and disease prognosis in NET patients. In the CLARINET study, $45 \%$ of patients had a primary tumor site in the pancreas, and $36 \%$ had a primary tumor site in the midgut. Another important factor is the clinical grade of NET. In the CLARINET study, $30 \%$ of patients had grade 2 (Ki-67 of 3-10\%) NET [6]. On the other hand, the earlier PROMID study with octreotide only examined grade 1 NET patients. Finally, from a prognostic point of view, the hepatic tumor volume is important. In the CLARINET study, in $33 \%$ of patients the hepatic tumor volume exceeded $25 \%[5,6]$.

\section{Results of the CLARINET study}

1. Progression-free survival (PFS) (primary endpoint) was significantly prolonged in patients receiving lanreotide. In the group receiving lanreotide (120 mg once every 4 weeks), the risk of disease progression or death was reduced by $53 \%$ compared to placebo. The median PFS had not been reached in patients receiving lanreotide, which means that it exceeded the 24-month study period. In the placebo group, the median PFS was 18 months. After 24 months, $65 \%$ of patients receiving lanreotide and 33\% of patients in the placebo group had achieved PFS [6].

2. The hazard ratio (HR) was $\mathbf{0 . 4 7}$ comparing the lanreotide group vs placebo. This means that patients treated with lanreotide showed a reduction in the risk of disease progression or death by 53\%. As shown in Fig. 1, $120 \mathrm{mg}$ of lanreotide once every 4 weeks has a clinical benefit in midgut NET, pancreatic NET and NETs of unknown origin. On the other hand, the HR was greater than 1 for hindgut NET, indicating an inconclusive result [6]. This may be consistent with a various clinical response in those tumors. Indeed, hindgut tumors do not usually express somatostatin receptors, they do not have elevated chromogranin A con-

Table 1 . Selected characteristics of the studied patients and the placebo group in the CLARINET study (modified according to 6 )

\begin{tabular}{|c|c|c|}
\hline & $\begin{array}{l}\text { Lanreotide (101 } \\
\text { patients) }\end{array}$ & Placebo (103 patients) \\
\hline Mean age, years (SD) & $63.3(9.8)$ & $62.2(11.1)$ \\
\hline Time (months) to diagnosis mean (SD) median & $32.6(46.1) 13.2$ & $34.4(41.4) 16.5$ \\
\hline Number of patients after resection of the primary tumor (\%) & $40(40)$ & $39(38)$ \\
\hline $\begin{array}{l}\text { Origin of neuroendocrine tumors (\%) } \\
\text { Pancreas } \\
\text { Midgut } \\
\text { Hindgut } \\
\text { Unknown/other }\end{array}$ & $\begin{array}{l}42(42) \\
33(33) \\
11(11) \\
15(15)\end{array}$ & $\begin{array}{c}49(48) \\
40(39) \\
3(3) \\
11(11)\end{array}$ \\
\hline $\begin{array}{l}\text { Tumor stage, number of patients }(\%) \\
1 \text { (Ki-67: 0-2\%) } \\
2 \text { (Ki-67: 3-10\%) } \\
\text { Unknown }\end{array}$ & $\begin{array}{c}69(68) \\
32(32) \\
0\end{array}$ & $\begin{array}{c}72(70) \\
29(28) \\
2(2)\end{array}$ \\
\hline $\begin{array}{l}\text { Degree of liver involvement, number of patients }(\%) \\
\begin{array}{l}0 \% \\
>0-10 \% \\
>10-25 \% \\
>25-50 \% \\
>50 \%\end{array}\end{array}$ & $\begin{array}{l}16(16) \\
33(33) \\
13(13) \\
23(23) \\
16(16)\end{array}$ & $\begin{array}{l}18(17) \\
40(39) \\
17(17) \\
12(12) \\
16(16)\end{array}$ \\
\hline $\begin{array}{l}\text { Chromogranin A, number of patients (\%) } \\
\leq 1 \times \text { upper limit of normal } \\
1-2 \times \text { upper limit of normal } \\
\leq 2 \times \text { upper limit of normal } \\
\text { Unknown }\end{array}$ & $\begin{array}{l}33(33) \\
25(25) \\
41(41) \\
2(2)\end{array}$ & $\begin{array}{l}34(33) \\
18(17) \\
48(47) \\
3(3)\end{array}$ \\
\hline
\end{tabular}


Table 2. Comparison of PROMID and CLARINET studies, as main studies of antiproliferative activity of SSAs $[5,6]$

\begin{tabular}{|c|c|c|}
\hline & CLARINET & PROMID \\
\hline Countries & international study: 14 countries & national study: Germany \\
\hline Randomized & 204 patients & $\begin{array}{l}\text { planned to recruit } 162 \text { patients } \\
85 \text { patients for interim analysis }\end{array}$ \\
\hline Tumor origin & $\begin{array}{l}\text { pancreas } \\
\text { midgut } \\
\text { hindgut } \\
\text { unknown }\end{array}$ & $\begin{array}{l}\text { midgut } \\
\text { unknown }\end{array}$ \\
\hline Functioning status & non-functioning only & $\begin{array}{l}\text { functioning (mild) and } \\
\text { non-functioning }\end{array}$ \\
\hline $\begin{array}{l}\text { Treatment status at } \\
\text { enrollment }\end{array}$ & $\begin{array}{l}\text { treatment-naïve and } \\
\text { post-surgery }\end{array}$ & $\begin{array}{l}\text { treatment-naïve and } \\
\text { post-surgery }\end{array}$ \\
\hline Progressive status & documented & unknown \\
\hline $\begin{array}{l}\text { Staging } \\
\text { (WHO classification) }\end{array}$ & $\begin{array}{l}\text { locally inoperable or metastatic } \\
\text { well or moderately differentiated }\end{array}$ & $\begin{array}{l}\text { locally inoperable or metastatic } \\
\text { well differentiated }\end{array}$ \\
\hline Ki-67 Index & $<10 \%$ & $<2 \%$ (for $95 \%$ of patients) \\
\hline Design & double-blind placebo-controlled & double-blind placebo-controlled \\
\hline Dose & lanreotide $120 \mathrm{mg}$ every 4 weeks vs placebo & $\begin{array}{l}\text { octreotide highest dose: } \\
30 \text { mg every } 4 \text { weeks vs placebo }\end{array}$ \\
\hline Treatment duration & $\begin{array}{l}96 \text { weeks } \\
\text { or until disease progression or death }\end{array}$ & $\begin{array}{l}18 \text { months } \\
\text { or until tumor progression or death }\end{array}$ \\
\hline Follow-up & not specified & on a yearly basis until death \\
\hline Primary endpoint & $\begin{array}{l}\text { time to disease progression } \\
\text { (according to RECIST) } \\
\text { or death, occurring within } 96 \text { weeks of first } \\
\text { injection }\end{array}$ & $\begin{array}{l}\text { time to tumor progression } \\
\text { (according to WHO criteria) }\end{array}$ \\
\hline Secondary endpoints & $\begin{array}{l}\text { patients without disease progression or death at } \\
48 \text { and } 96 \text { weeks } \\
\text { time to progression } \\
\text { overall survival } \\
\text { quality of life } \\
\text { chromogranin A } \\
\& \text { other tumor markers } \\
\text { safety of lanreotide } \\
\text { pharmacokinetics of lanreotide }\end{array}$ & $\begin{array}{l}\text { tumor response at } 6 \text { months } \\
\text { survival time } \\
\text { quality of life } \\
\text { chromogranin A } \\
\text { symptomatic response } \\
\text { safety of octreotide }\end{array}$ \\
\hline
\end{tabular}

centrations, and they often do not respond to SSA administration [7] (Fig. 1).

3. The median PFS was lengthened in all subgroups treated with lanreotide. The therapeutic effect of lanreotide in each selected group was consistent with the effect in the general population as follows:

In the subgroup of patients with midgut NET the median PFS exceeded the 24-month study period. In the placebo group, the median PFS was 21 months.

More events were reported in the placebo group than in patients receiving lanreotide.

In patients with pancreatic NET the median PFS exceeded the 24-month period of the study. In the placebo group the median PFS was 12 months, which means that more events were reported in the placebo group than in patients treated with lanreotide.

In patients with grade 1 and 2 (G1 or G2) NET, the median PFS exceeded the 24-month study period in both subgroups. The median PFS in the placebo group was 18 months in patients with G1 NET and 12 months in patients with G2 NET.

Treatment of patients with lanreotide was effective regardless of hepatic tumor volume. In the subgroup with hepatic tumor volume $<25 \%$, the median PFS exceeded the 24-month study period for the group receiving lanreotide, and in the placebo group the median PFS was 21 months. In the subgroup with hepatic tumor volume $>25 \%$, the median PFS exceeded 24 months for the group receiving lanreotide compared to the placebo group, where it was 9 months [6].

The long PFS in the placebo groups gives clinicians a period of observation. The placebo group may be considered a surrogate for deferred treatment or the 'wait and watch' strategy [7]. This observation period enables clinicians to assess the tumor's biology and grade, and determine the optimal time to introduce therapy. This strategy was ad- 


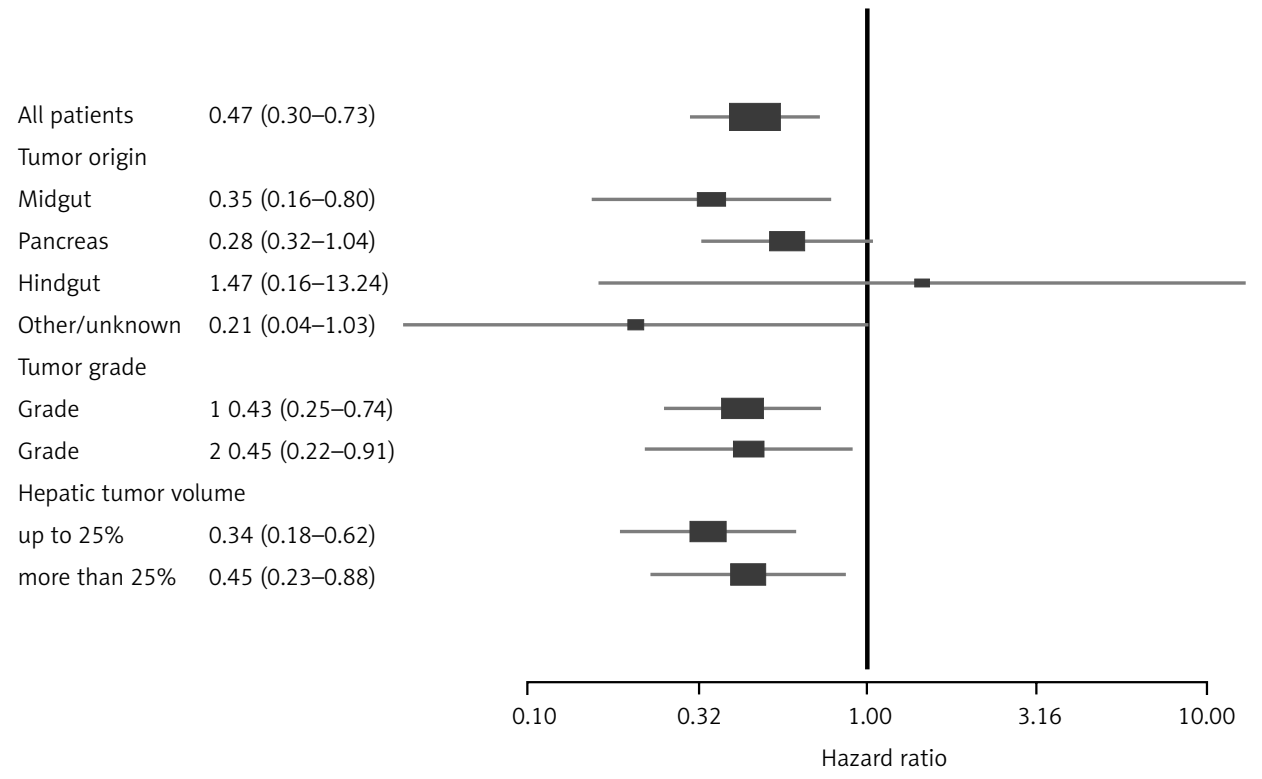

Fig. 1. Hazard ratios (HR) for patients with NET taking either lanreotide or placebo in the overall population of patients. Subgroups are selected according to primary tumor site, stage of NET and the degree of liver involvement (modified according to [6])

vised in order to delay the onset of unwanted side effects related to particular NET treatment methods. The 'wait and watch' method also prevents the hasty introduction of NET therapies, which may exhaust all treatment possibilities too quickly. However, progression is significantly delayed in the group receiving lanreotide, so the 'wait and watch' strategy could be considered in selected cases.

4. No significant differences in overall survival were observed. The overall survival was similar in both groups (lanreotide and placebo), which is probably due to the long life expectancy of patients with slowly progressive tumors. However, the analysis of overall survival is complicated by cases where patients switched groups (i.e., patients switched from the placebo group to the group receiving lanreotide after disease progression) [6].

5. No significant differences in quality of life were observed. Treatment with lanreotide did not adversely affect patients' quality of life [6]. Therefore, this is a useful and safe therapeutic method, especially compared to other available NET pharmacotherapies, which have a number of side effects.

6. Chromogranin A ( $\mathrm{CgA}$ ) concentrations were reduced in the lanreotide group. CgA concentrations are considered an important prognostic factor in NET. In patients with a baseline CgA concentration above the upper limit of the normal range, the odds ratio to reduce its concentration by $\geq 50 \%$ was significantly higher in patients receiving lanreotide than in the placebo group [5]. This is particularly important because high concentrations of CgA negatively correlate with survival of patients with NET $[1,2]$.

7. Lanreotide has a good safety profile. In the lanreotide treated group, half of NET patients experienced adverse reactions. However, $28 \%$ of the placebo group also experienced adverse events. The most common adverse event associated with treatment was diarrhea ( $26 \%$ of pa- tients receiving lanreotide and $9 \%$ of patients in the placebo group) [6].

\section{Summary of significance for clinical practice}

1. The CLARINET study provided important information on the use of lanreotide in clinical practice for controlling tumor growth control in patients with advanced GEP NETs. While the previous PROMID clinical trial indicated the beneficial anti-cancer effects of octreotide, this study failed to include patients with NETs of the pancreas or G2 NETs, which are frequently encountered in clinical practice. Therefore, the CLARINET study empowers clinicians to use the anti-proliferative effect of lanreotide to a much broader extent, i.e., in patients with highly differentiated NET, with different primary tumor locations.

2. Considering the results of the CLARINET study, the conservative approach of observation ('wait and watch') should be re-analyzed. This option should be left only for individual NET cases or situations where the clinical trials did not provide sufficient evidence regarding benefits of its application (Table 2).

3. The results of the CLARINET clinical trial indicate that the progression-free survival (PFS) of patients with metastatic and unresectable GEP NET G1 and G2 (Ki-67 $<10 \%$ ), regardless of the degree of liver involvement, who received lanreotide at a dose of 120 mg every 4 weeks was significantly prolonged in cases of stable disease course. The study shows a significant effect of treatment on prolongation of PFS, but without significant differences in the OS. A recent observational study suggested a link between prolongation of PFS and OS in a large cohort of patients with metastatic NET treated with somatostatin analogs. These findings support the use of PFS as an important endpoint in NET clinical trials [9]. 
4. An important observation in the CLARINET study was the favorable tolerability and safety of lanreotide.

These data support the possible inclusion of this SSA at an early stage of NET management, as already suggested by many organizations and scientific societies [1, 10, 11].

The author declares no conflict of interest. Ipsen Poland provided support for medical writing translation.

\section{References}

1. Kos-Kudła B, Blicharz-Dorniak J, Handkiewicz-Junak D, et al. Diagnostic and therapeutic guidelines for gastro-entero-pancreatic neuroendocrine neoplasms (recommended by the Polish Network of Neuroendocrine Tumours). Endokrynol Pol 2013; 64: 418-43.

2. Bolanowski M, Bednarczuk T, Bobek-Billewicz B, et al. Neuroendocrine neoplasms of the small intestine and the appendix - management guidelines (recommended by the Polish Network of Neuroendocrine Tumours). Endokrynol Pol 2013; 64: 480-93.

3. Rosiek V, Kunikowska J, Kos-Kudła B. A non-functioning pancreatic neuroendocrine tumour: a case report. Endokrynol Pol 2012; 63: 59-64.

4. Wolin E, Hu K, Hughes G, et al. Safety, tolerability, pharmacokinetics, and pharmacodynamics of a long-acting release (LAR) formulation of pasireotide (SOM230) in patients with gastroenteropancreatic neuroendocrine tumors: results from a randomized, multicenter, open-label, phase I study. Cancer Chemother Pharmacol 2013; 72: 387-395.

5. Rinke A, Muller HH, Schade-Brittinger C, et al. Placebo-controlled, double-blind, prospective, randomized study on the effect of octreotide $L A R$ in the control of tumor growth in patients with metastatic neuroendocrine midgut tumors: a report from the PROMID Study Group. J Clin Oncol 2009; 27: 4656-63.

6. Caplin M, Pavel M, Ćwikła JB, et al. Lanreotide in metastatic enteropancreatic neuroendocrine tumors. New Engl J Med 2014; 371: 224-33.

7. Starzyńska T, Deptała A, Królicki L, et al. Colorectal neuroendocrine neoplasms - management guidelines (recommended by the Polish Network of Neuroendocrine Tumours). Endokrynol Pol 2013; 64: 494-504.

8. Garcia-Carbonero R, Jimenez-Fonseca P, Teulé A, et al. SEOM clinical guidelines for the diagnosis and treatment of gastroenteropancreatic neuroendocrine neoplasms (GEP-NENs) 2014. Clin Transl Oncol 2014; 16: 1025-34.

9. Ter-Minassian M, Brooks NV, Brais LK, et al. Association of progression-free survival with overall survival (OS) in patients (pts) with neuroendocrine tumor (NET) treated with somatostatin analogs. J Clin Oncol 2015; 33 (suppl): abstr 4090.

10. Pavel M, Baudin E, Couvelard A, et al. ENETS Consensus Guidelines for the management of patients with liver and other distant metastases from neuroendocrine neoplasms of foregut, midgut, hindgut, and unknown primary. Neuroendocrinology 2012; 95 : 157-76.

11. Kulke $M H$, Shah $M H$, Benson $A B$, et al. Neuroendocrine Tumors, Version 1.2015. J Natl Compr Canc Netw 2015; 13: 78-108.

\section{Address for correspondence}

\section{Beata Kos-Kudła}

Division of Endocrinology

Department of Pathophysiology and Endocrinology Medical University of Silesia

Ceglana 35

40-952 Katowice, Poland

e-mail: bkoskudla@sum.edu.pl

Submitted: 21.08 .2015

Accepted: 24.11.2015 